Int. J. Dev. Biol. 48: 1087-1094 (2004)

doi: $10.1387 / \mathrm{ijdb} .041859 \mathrm{am}$

Original Article

\title{
PVF1/PVR signaling and apoptosis promotes the rotation and dorsal closure of the Drosophila male terminalia
}

\author{
ANA MACÍAS ${ }^{1 *}$, NURIA M. ROMERO², FRANCISCO MARTÍN ${ }^{3}$ LEONARDO SUÁREZ1 , ALBERTO L. ROSA² \\ and GINÉS MORATA ${ }^{3}$
}

\begin{abstract}
${ }^{1}$ Laboratorio Genética del Desarrollo, FCEF y $N$ de la Universidad Nacional de Córdoba, Argentina, ${ }^{2}$ Laboratorio de Neurogenética, Instituto
\end{abstract} de Investigación Médica "Mercedes y Martín Ferreyra", INIMEC-CONICET, Argentina and ${ }^{3}$ Centro de Biología Molecular, UAM, CSIC, Spain

\begin{abstract}
The Drosophila adult male terminalia originate from the genital disc. During the pupal stages, the external parts of terminalia evert from two ventral stalks; the everted left and right dorsal halves fuse at the dorsal midline. At the same time the male terminalia perform a $360^{\circ}$ clockwise rotation. Several mutations are known to affect the rotation of the male terminalia, while none is known to affect dorsal closure. We show here that the Pvf1 gene, encoding one of the three Drosophila homologues of the mammalian VEGF/PDGF growth factors, is required for both processes. Males either mutant for Pvf1 or bearing a dominant negative form of Pvr or stasis (stai), the unique PVF receptor, do not complete either rotation or dorsal closure. Pvf1 expression in the genital disc is restricted to the A8 cells. However, PVF1/PVR signaling influences A8, A9 and A10 cells, suggesting that the PVF1 protein diffuses from its source. Flies hemizygous for the apoptotic genes hid, reaper and grim, or mutant for puckered which encodes a phosphatase that downregulates the $n-J u n-N$ terminal kinase pathway, lead to the same phenotypes as mutations in PVF1/ PVR. Our results indicate that PVF1/PVR signaling functions not only in apoptotic phenomena but are also required during rotation and dorsal closure of the Drosophila male genital disc.
\end{abstract}

KEY WORDS: PVF1/PVR, VEGF/PDGF, apoptosis, Drosophila, male genital disc

\section{Introduction}

The adults terminalia of Drosophila derive from the genital disc (reviewed in Sánchez and Guerrero, 2001). It is constituted by the fusion of three embryonic abdominal segments: A8, A9 and A10. In the male genital disc the $A 8$ primordium gives rise to a tiny $A 8$ tergite (T8), whereas the $A 9$ forms the male genitalia and $A 10$ the male analia and hindgut. The shaping process of these structures occurs during the pupal stages; among them there is the fusion in the dorsal midline of left and right halves of this bilateral symmetrical disc and a $360^{\circ}$ clockwise rotation (Gleichauf, 1936; Adám et al., 2003) (see Fig. $1 \mathrm{~A}, \mathrm{~B}$ ) related with the maturation of the internal genitalia (Gleichauf, 1936) (see Fig. 1C). No mutations are known to affect dorsal closure, but several have been reported to affect the rotation of the male terminalia. The latter include mutations at Abdominal-B $(A b d-B)$ (Casanova et al., 1986; Sánchez-Herrero and Crosby, 1988), head involution defective (hid) (Abbott and Lengyel, 1991; Grether et al., 1995), at the gene encoding transcription factor TAF250 (Wassarman et al., 2000), spin (Adám et al., 2003) and at some unidentified genes located in the region 11A (Fahmy and Fahmy, 1958). Most of those mutants present several degrees of incomplete rotation. The involvement of hid is of interest, for it is a pro-apoptotic gene (Grether et al., 1995) and suggests that cell death is required.

In Drosophila, the PVF/PVR signaling pathway has been involved in border cell migration in the oocyte (Duchek et al., 2001, McDonald et al., 2003) as well as in migration-survival and proliferation of the hemocytes in embryos and larvae (Heino et al., 2001; Cho et al., 2002, Munier et al., 2002; Brückner et al., 2004).

There are three Pvfs genes in Drosophila. The mammalian homologues of these ligands could form homo or heterodimers, the type of dimer formed specifies the response of the receptor (Heldin and Westermark, 1999). On the contrary, there is only one receptor for PVFs, Pvr o stasis (stai), with demonstrated binding activity to PVF1 (Duchek et al., 2001).

Abbreviations used in this paper: JNK, n-Jun-N terminal kinase; PDGF, platelet derived growth factor; puc, puckered gene; PVF1, platelet vascular factor1; VEGF, vascular endothelial growth factor; WT, wild-type.

\footnotetext{
*Address correspondence to: Dra. Ana Macías F.C.E.F y N. de la U.N.C. Av.Vélez Sarsfield 299. 5000 Córdoba, Argentina. Fax: 54-351-433-2097. e-mail: amacias@com.uncor.edu
} 
In this work we show that PVF1/PVR signaling is involved in dorsal closure and rotation of male terminalia, as mutations in genes encoding the ligand or in the receptor alter these processes. We show that Pvf1 transcript is only expressed in part of $A 8$ of male genital discs but is required in cells of the three segments, suggesting a diffusion of the product. We also confirm the role of hidin terminalia rotation (Abbott and Lengyel, 1991) and show that blocking cell death by the baculovirus caspase inhibitor p35, or altering the apoptotic n-Jun-N terminal Kinase pathway produce the same phenotype as hid - and the lack of function of PVF1/PVR.

\section{Results}

\section{Genetic analysis of the Pvf1 gene}

To identify genes involved in the development of adult male terminalia we analyzed P-Gal4 lines using the yellowmethod (Calleja et al., 1996). The line LP23Gal4 was selected and characterized in detail. Inverse and direct PCR, as well as Southern blot analysis, showed that the insertion is located 250 bp upstream the proposed transcription start site of the Pvf1 gene (Fig. 2; Duchek et al., 2001, Cho et al., 2002). The expression pattern driven by LP23-Gal4 in embryos corresponds to a subset of Pvf1 expressing cells previously described (Cho et al., 2002; data not shown). LP23-Gal4 males have wild type terminalia. The specific $y+$ adult expression in the male terminalia suggested a possible function of $P v f 1$ in this region of the body. To characterize its function the LP23 insertion was mobilized to generate deficiencies after imprecise jumps (Gloor et al., 1991). Six putative imprecise excisions were semilethal in males and showed adult male rotated terminalia (Fig. 3A). The $D p(1 ; Y)$ W39 (Prado et al., 1999) rescued these phenotype. Since this duplication includes the wild type allele of $P v f 1$, this result suggested that the phenotype observed in the excised LP23-Gal4 lines were probably due to a defective Pvf1 gene. Detailed Southern blot and PCR analyses were performed for two of these mutants, $\angle P 23^{\text {semil/ }}$ and $\angle P 23^{\text {semil2 }}$ (Fig. 2). It was found that $\angle P 23^{\text {semil1 }}$ is a deletion that removes part of the 5 ' region upstream the transcription starts site (Duchek et al., 2001; Cho et al., 2002), the first exon and part of the first intron of the Pvf1gene,

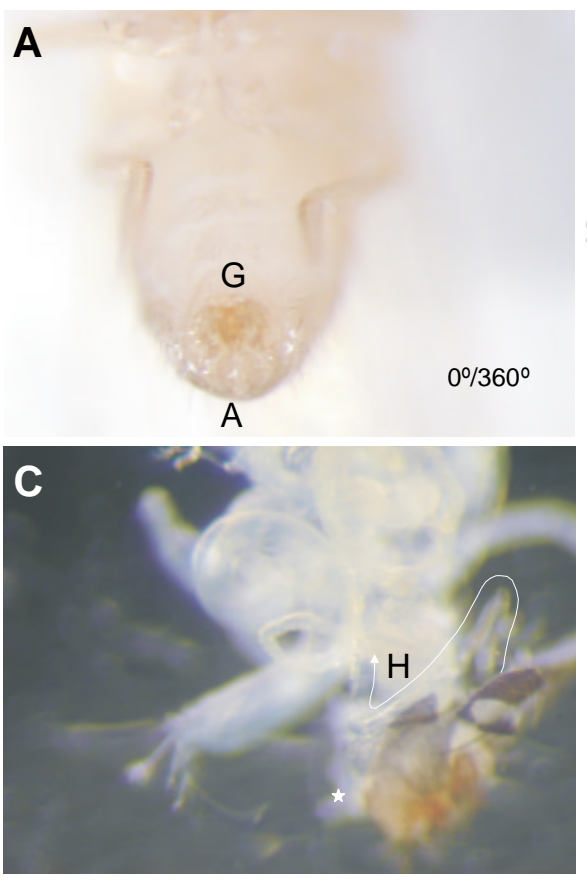

B

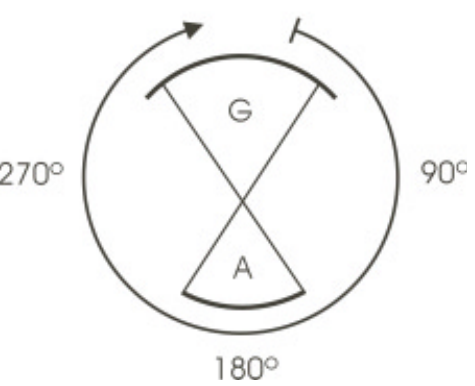

Fig. 1. Wild type adult male terminalias. (A) A wt male with its ventral side up, showing the correct position of external terminalia. In all the figures the position of the genitalia is indicated by $G$ and the analia by $A$; the degree of rotation reached is expressed in the lower right angle. (B) Schematic representation showing the direction and degree of rotation, ventral corresponds to the upper half. (C) Dissection, showing the turns of the spermiduct around the hindgut (H). The white line indicates the $360^{\circ}$ spiral trajectory of the spermiduct; the white star dicates the sperm pump location on the right side of the organism.

as well as most of the P-Gal4 transposon (Fig. 2). $\angle P 23^{\text {semil/2 }}$ is also a deletion with a breakpoint in the first intron of Pvf1, extending 5' more distally than $\angle P 23$ semilt, removing the transposon and deleting the neighboring gene CG7101 (Fig. 2). We also analyzed the mutant Pvf1 ${ }^{1624}$, carrying the transposon EP1624 (Rørth, 1996) inserted in the first intron of the Pvf1 gene (Duchek et al., 2001). This allele is homozygous viable (Duchek et al., 2001), albeit there is some pupal lethality and the adults show reduced fertility (not shown). Hemizygous Df (1)LP23 semill and PVf1'1624 males show a similar rotated terminalia (Fig. 3 A,B). The phenotype is not fully penetrant and the expressivity is variable, but both increase at higher temperatures (Fig. $3 \mathrm{D}, \mathrm{E}$ ). Occasionally, we noticed a lack of dorsal closure in some individuals (not shown). The variability of the phenotype could be due to a partial redundancy in Pvfgenes function, as it has been described for other Pvf associated phenotypes (Ducheck et al., 2001; Cho et al., 2002.)

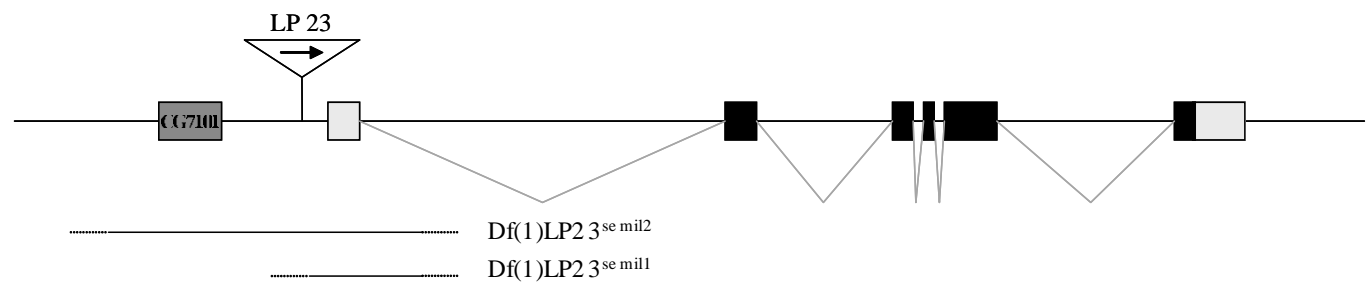

Fig. 2. Structure and product of the Drosophila Pvf1 gene. The Pvf1 locus at cytological position 17E. The open reading frame (ORF) of the transcript is indicated as black boxes. The arrow on $P$ transposon $p$ Gaw $\beta$, called LP23 indicates the direction of Gal4-induced transcription. The sequences deleted in Pvf1, i.e. Df(1)LP23 $3^{\text {semil1 }}$ and Df(1)LP23 $3^{\text {semil2 }}$ are indicated; dotted extensions indiPVF1 PDGF domain cate the range of uncertainty in deletion endpoints. 


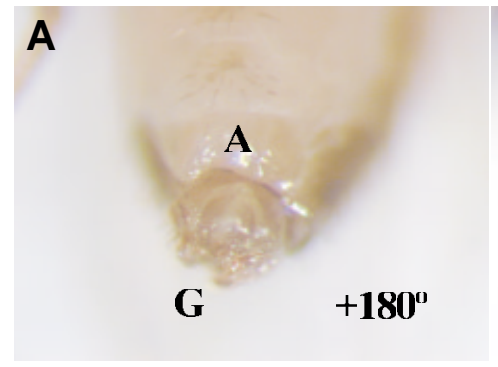

B

D

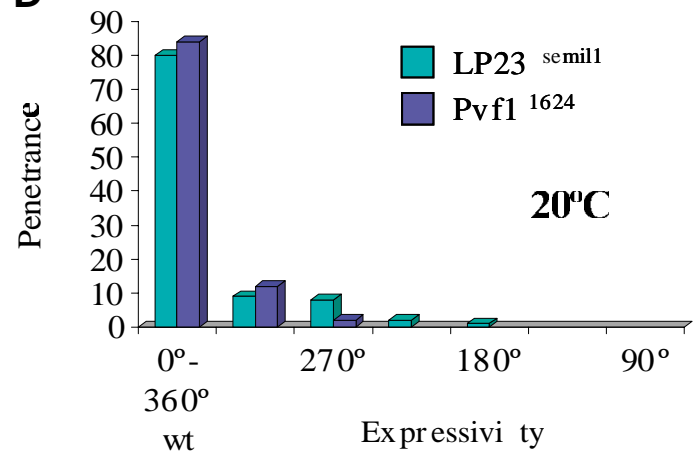

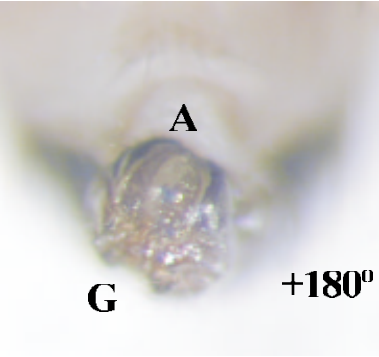

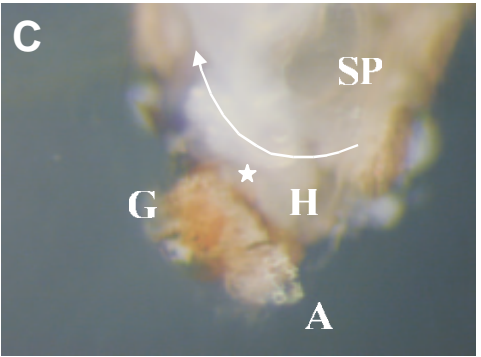

E

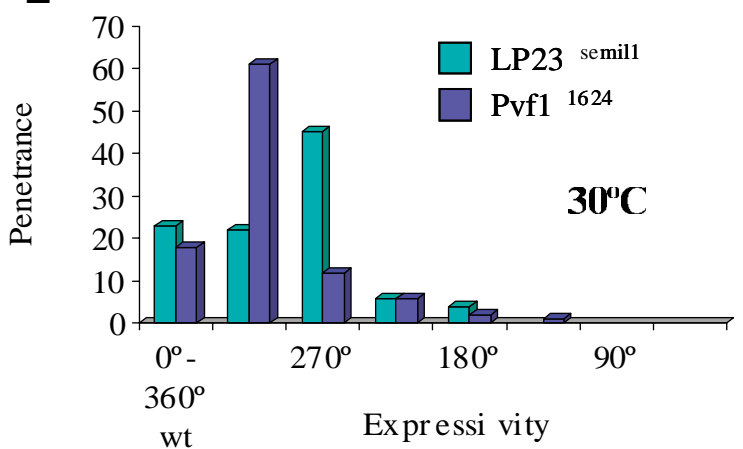

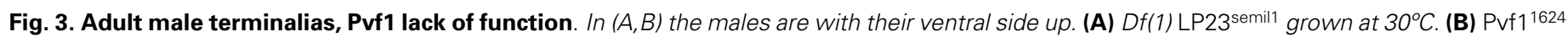

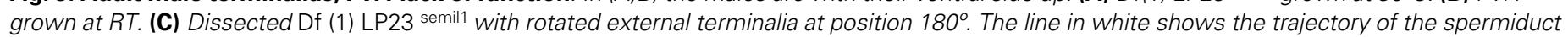

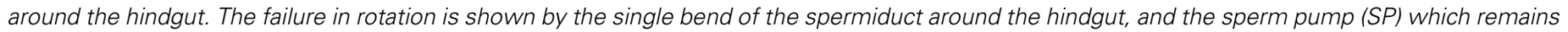

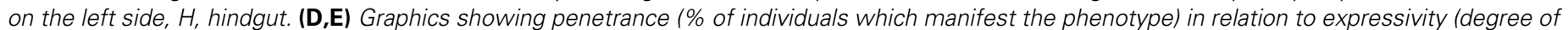
rotation) of Pvf1 mutants at different temperatures; the rotated phenotype is temperature sensitive. (D) Grown at $20^{\circ} \mathrm{C}$. (E) Grown at $30^{\circ} \mathrm{C}$.

The analyses of terminalia phenotypes are easily performed by microscope observation of the spermiduct trajectory and the sperm pump position (Fig. 1C). In wild type males, the spermiduct bends twice around the hindgut and the sperm pump is located in the right side of the abdomen (Fig. 1C). The internal analyses of Pvf1 mutant flies, with various degrees of rotated terminalia, showed that the direction of rotation is not altered in the mutants (Figs. 3C, 1C). The spermiduct normally initiated a clockwise- turn but stops prematurely, while the sperm pump that is on the right side, is on the left in the mutants. All the external structures of the terminalia appear to move coordinately with the internal spermiduct and sperm pump during rotation, because all of them are misspositioned in a equivalent rotated stage when the movement fail to be completed.

\section{Pvf1 expression in the male genital disc}

Pvf1 transcripts are first detected in late third instar larvae, in cells that belong to A8 segment (Fig. 4 A,B). We delimited Pvf1 expression using as a reference the engrailed (en), decapentaplegic (dpp) and patched (ptc) (Fig. 4 C,D) genes. ptc expression is closely associated to that of enin the genital disc (Fig. 4D), while $d p p$ is more restricted and co-expresses with enonly in few cells (Fig. 4C). In A8, Pvf1 expression may overlap that of $e n, d p p$ and ptc only in few cells indicated in Fig. 4 C,D. The Pvf1 expression domain extends to a group of anterior cells, outside the A8 ptcl dppdomain and corresponds to two bands of cells -left and right- that occupy the central region of the segment $A 8$ separated by an intermediate zone that does not express the gene. Since PVF1/PVR activates the RAS-MAPK pathway (Duchek et al., 2001, Cho et al., 2002, Brückner et al., 2004)), PVF1/PVR activity can be detected with an antibody that reveals active ERK, (anti- dpERK) (Gabay et al., 1997). In the wild type male genital disc dpERK could be detected only in scattered cells of A8 (Fig. 4E). However, increasing the amount of the receptor PVR (e.g. using ptc-Gal4; UAS-PVr) dpERK activity was detected in the periphery of $P v f 1$ transcript expression, in the ptc domain of A8 and a subset of ptcA9 cells in the vicinity (Fig. 4F). This observation indicates that PVF1 activity extends further to its expression domain.

\section{Inactivation of the Pvf receptor}

We extended the study of the role of Pvrfunction by expressing in restricted domains a dominant negative form, $P v r^{D N}$ (Duchek et al., 2001), using the Gal4/UAS system (Brand and Perrimon, 1993). In these studies we used drivers expressed in the posterior ( $h$ h-Gal4, en-Gal4) or in the anterior compartment (ptc-Gal4, dppGal4) (Fig. 4 C,D). The activity of $P v r D N$ results in a phenotype of rotated terminalia (Fig. $5 \mathrm{~A}, \mathrm{~B}$ ), which is stronger with en-Gal4 (Fig. 5D) and similar to that observed in flies carrying mutations in Pvf1 (Fig. $3 \mathrm{~A}, \mathrm{~B}$ ). The penetrance and expressivity of this phenotype was variable, suggesting that some residual $P v r$ function was present; sometimes, as observed with Pvf1 mutants, the dorsal closure was not completed.

We tested if there is a more extreme phenotype in males Pvf1;en-Gal4; UAS- PVrDN. All these males have the terminalia rotated between $180^{\circ}$ and $270^{\circ}$ and show the dorsal split phenotype (not shown). This confirms the specificity of PVF1 for PVR (Duchek et al., 2001). The observation of the internal spermiduct and sperm pump structures in these flies is similar to that observed in the Pvf1 mutants (Fig. 5C). 

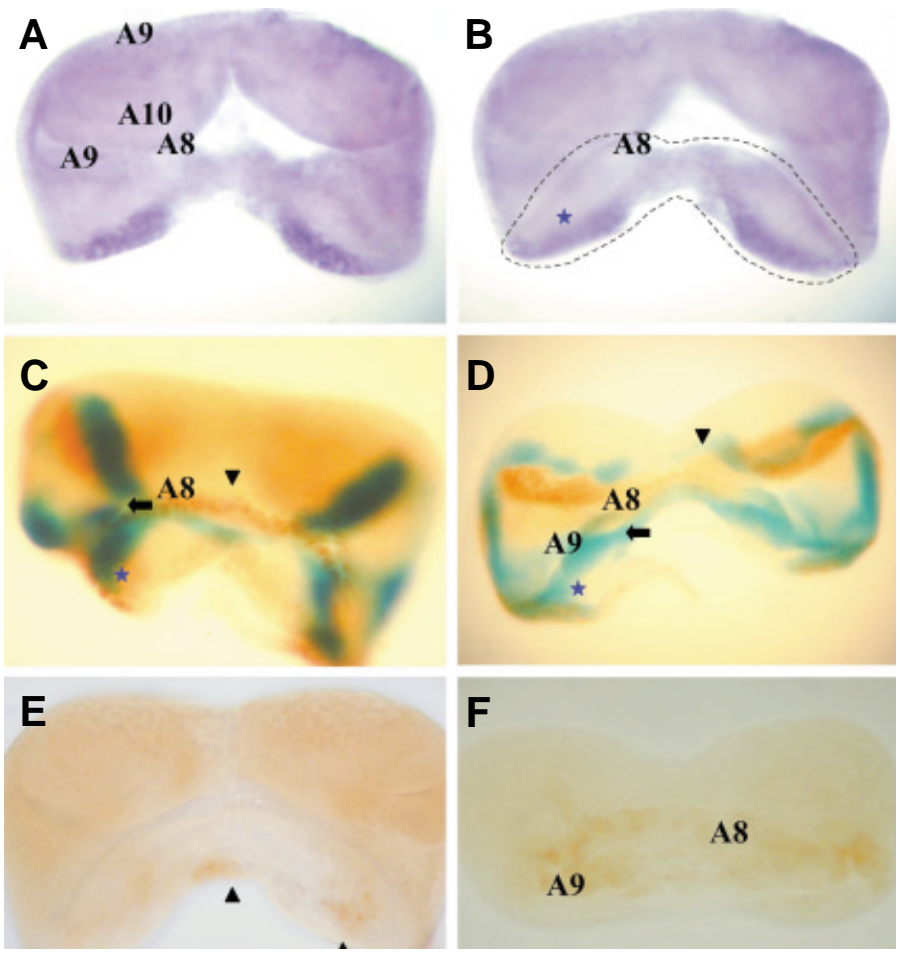

Fig. 4. Pvf1 expression in wild type male genital discs. $(A, B)$ Pvf1 transcript expression. (A) The dorsal side is in focus; the regions corresponding to the different abdominal segment cells are indicated. (B) The ventral side is in focus; the dotted line border outlines the A8 segment; the blue star indicates the region of probable overlap between Pvf1, en, dpp and ptc expression. (C,D) Domains of expression of the pattern genes en, $\mathrm{dpp}$ and ptc. Gal 4 insertions in these genes were used to direct targeted gene expression. The ventral side is in focus, revealing the position of the A8 segment. The blue stars indicate the same region as in (B). The arrows indicate dpp or ptc expression, while the arrowhead indicates en expression. (C) The expression of dpp (blue) is revealed using the dpp-lacZ transgene (arrow) and the expression of EN (brown-orange) is revealed using an anti-EN antibody (arrowhead). In some areas, dpp follows EN expression. (D) ptc expression in blue revealed by the ptc-lacZ transgene (arrow); EN expression in brown-orange, was revealed with an anti-EN antibody. Note that ptc expression is parallel to that of en (arrowhead). (E) A wt disc showing the presence of activated ERK (brown) by means of an anti-dpERK antibody (arrowheads). (F) ptc-Gal4; UAS-Pvr, the cells in brown are positive for dpERK; these cells correspond to the ptc domain of A8 and part of A9 cells. See ptc expression in (C).

Taken together these results show that lack of $P v f 1$ or inactivation of its receptor causes similar phenotypes, so the PVF1/PVR signaling is required. Remarkably, blockage of the signaling mechanism is most sensitive in the engrailed domain (Fig. 5D), where Pvf1 transcript was not detected.

\section{Pvf1 gain of function}

The EP11235 line (Rørth, 1998) has a gain of function allele for $P v f 1$ as the EP element is inserted upstream of the Pvf1 transcription start site (Duchek et al., 2001). With the $d p p$-Gal4 or ptc-Gal4 drivers EP11235 overexpression has no phenotypic effects, but with en-Gal4 or $h h$-Gal4 it does (Fig. 5E). Increasing PVF1 levels in males EP11235; en-Gal4:2xUAS-Pvf1 augmented the percent- age of miss-rotations and the split phenotype (Fig. 5F). As the $w t$ expression of $P v f 1$ does not overlap that of engrailedin the genital disc, this result supports the idea that the diffusion of PVF1 is instructive in terminalia rotation and dorsal closure.

\section{Apoptosis involved in dorsal closure and rotation}

hidis a pro-apoptotic gene (Grether et al., 1995). hidalleles are embryonic lethal and heterozygous individuals are wild type. Males escapers hidA22(Grether et al., 1995) over Df(3L)H99 (a deficiency that removes hid and the other pro-apoptotic genes reaper and grim (Grether et al., 1995; Chen et al., 1996; Chao and Nagoshi, 1999) reared at $25^{\circ} \mathrm{C}$ have the terminalia rotated between $90^{\circ}$ and $270^{\circ}$ (Abbott and Lengyel, 1991) (data not shown). Interestingly, $50 \%$ of heterozygous $\mathrm{Df}(3 \mathrm{~L}) \mathrm{H} 9 \mathrm{~g} /$ / males have a dominant phenotype of rotated terminalia when grown at $30^{\circ} \mathrm{C}$. To analyze whether Pvf1 and Df(3L)H99 interact in eliciting this phenotype we constructed males of genotypes Df(1)LP23 Pvff1624; Df(3L)H99/t. They showed a notable increase in penetrance, from $20 \%$ of $D f(1) L P 23^{\text {semil1 }}$ or $P V f 1^{1624}$ alone to $80 \%$ with the deficiency (Fig. $6 \mathrm{~A}, \mathrm{~B})$. This increment suggests an interaction between the pro-apoptotic genes included in Df(3L)H99and Pvfin the process of terminalia rotation. Since hidis a pro-apoptotic gene (Grether et al., 1995), we hypothesized that the rotated phenotype may be caused by and inhibition or reduction of apoptosis. To test this possibility, the gene encoding the caspase inhibitor p35 (Hay et al., 1994) was expressed in the male genital disc. We find that males of genotype en-Gal4; UAS-p35 and dpp-Gal4; UAS-p35 possess rotated terminalia and a gap in the dorsal midline (Fig. 6C). Driving the expression of $p 35$ to the posterior cells led to a stronger phenotypes than using anterior Gal4 drivers (not shown).

It has been shown that the activity of RAS-MAPK, one of the mayor signal transduction players of RTK (reviewed in Seger and Krebs, 1995), produces a decreased activity of hid (Kurada and White, 1998; Bergmann etal., 1998). Accordingly, we analyzed the consequence of Ras overexpression in the rotation of the male terminalia. A low percentage $(4 \%)$ of males carrying the UASRas $^{A V 12}$ transgene, a constitutive form of the RAS (Fontini et al., 1992), show at RT, a dominant phenotype of rotated terminalia (not shown). This effect may be based on a basal expression of the construct. However, expression of $\operatorname{Ras}^{\mathrm{AV} 12}$ at $30^{\circ} \mathrm{C}$ using the driver LP23-Gal4 led to a much higher percentage (55\%) of adult males with rotated terminalia (Fig. 6D). Therefore, overexpressing Rasin the male genital disc causes the same phenotype observed in the eye-antenna disc, as it is reduces hid activity (Kurada and White, 1998; Bergmann et al., 1998).

\section{Role of the JNK signalling pathway}

The JNK pathway is involved in apoptosis in the imaginal discs (Moreno et al., 2002 a). Furthermore, JNK, together with Eiger -the Drosophila TNF homologous- up-regulates hid (Moreno et al., 2002 b). Therefore, we hypothesized that alterations in this pathway could also affect terminalia rotation and dorsal closure.

JNK activity can be down regulated in Drosophilaby increasing the expression of the gene puckered (puc), an element of the pathway encoding a dual-specificity phosphatase that generates a negative feedback loop (Martín-Blanco et al., 1998). Targeted expression of UAS-pucin the genital disc using en-Gal4, dpp-Gal4, or ptc-Gal 4 causes rotation of the male terminalia and dorsal split, remarkably observed in the ptc-en domains (Fig. 7 B-E). In these 
A

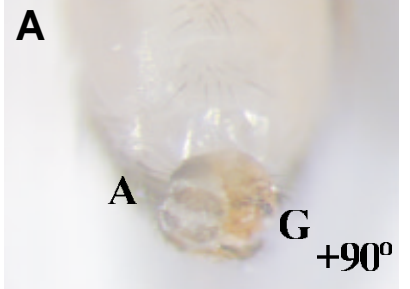

B

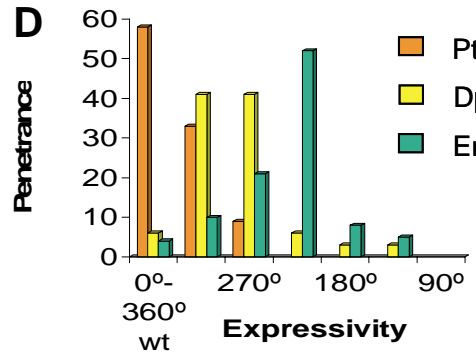

$\mathbf{F}$
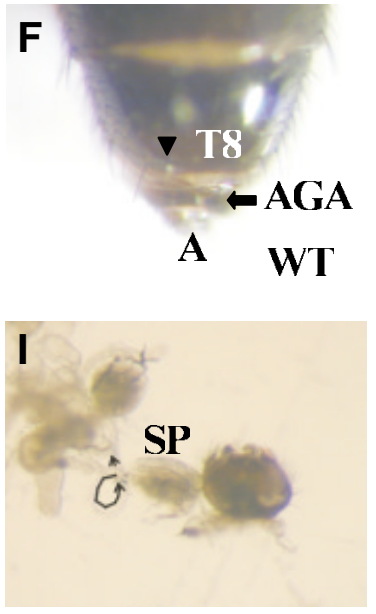

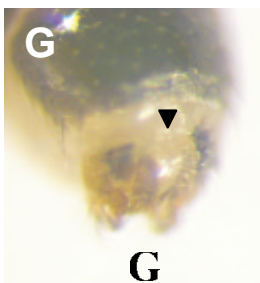

G

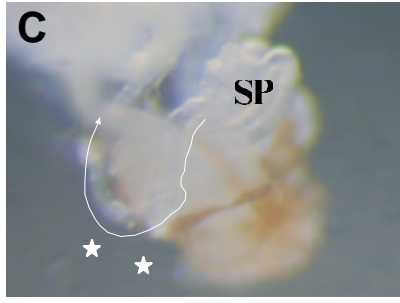

$\mathbf{E}$
A

$30^{\circ} \mathrm{C}$
Fig. 5. Adult male terminalia, Pvr lack of function and Pvf1 gain of function. The flies were grown at $30^{\circ} \mathrm{C}$. The males $A, B$ and $E$ are with its ventral is side up. (A) dpp-GAL4; UAS-PvrDN. (B) en-Gal4; UAS-PvrDN . (C) Dissecteden-Gal4;UAS$\mathrm{Pvr}^{\mathrm{DN}}$ male, the external terminalia was at $+270^{\circ}$, the white line show the trajectory of spermiduct, the duct could do two bends (white stars) but the sperm pump is found on the left. (D) Graphic penetrance/expressivity of PvrDN expression, the response to the receptor lack of function is stronger in the en domain. (E) en-Gal4:2x UAS-Pvf1,

overexpressing Pvf1 in the en domain produce rotated terminalia. (F) Wild type male with its dorsal side up to show the morphology of terminalia in these side, analia (A), anterior genital arch (AGA) (arrow), tergite 8 (T8)(arrowhead). (G) Dorsal view of hh-Gal4:EP11235, 2xUAS-Pvf1, to show the failure in dorsal closure. The tergite 8 (T8), the anterior genital arch and the dorsal anal plates do not fuse in the dorsal midline (arrowhead). (H) Genital disc of hh-Gal4; EP11235; 2x UAS-Pvf1 hybridized with Pvf1 RNA, showing the endogenous and the exogenous expression of Pvf1. (I) hh-Gal4; 3x UAS-Pvf1, the external terminalia was at $180^{\circ}$, the spermiduct is followed by a black line, the duct only does one bend, the sperm pump (SP) is on the left.

flies, the spermiduct and sperm pump are miss-rotated (Fig. 7D), as in Pvf1lPvr.

To further study the association between the JNK and the PVF1/ PVR pathways we examined JNK activity by checking the expression of puc-lacZtransgene in males either puc ${ }^{E 69}$ a lethal allele with the puc-lacZ insertion, or hh-Gal4; UAS-Pvf1/puc E69. It was observed that the expression domain of puc surrounds that of $P v f 1$ (Fig. 7F) and extends to A9 cells. Targeted expression of Pvf1 can ectopically activate puc(Fig. 7G). We also analyzed whether increasing the activity of JNK by lowering puc may rescue the dominant phenotype of $D f(3 L) H 99 /+$ males. We found that $100 \%$ of the males double heterozygous for $D f(3 L) H 99$ and $p u c^{E 69}$ are wild type at $30^{\circ} \mathrm{C}$, whereas $50 \%$ of $D f(3 L) H 99 /+$ males have a mutant phenotype. This result strongly supports the idea that in the genital disc the JNK pathway is also activating hid, as it has already been demonstrated in other genetic context (Moreno et al., 2002 b).

\section{Discussion}

The PVF1/PVR pathway and the rotation and dorsal closure of male terminalia

In this work we demonstrate that either mutations at $P v f 1$ or the expression of a dominant negative form of its unique receptor, PVPN , result in various degrees of male rotated terminalia and failure of dorsal closure. These observations indicate that the PVF1/PVR pathway is relevant in these morphogenetic processes. Although the Pvf1 gene is only expressed in a subset of cells from the segment $A 8$, reduction or abolition of PVF1/PVR signaling affects the normal development of all terminalia precursors (A8, A9 and $\mathrm{A} 10)$. Interestingly, mutations in the $A b d-B m$ function, which affect only the A8 segment (Casanova et al., 1986) have a phenotype of rotated terminalia. Thus, these results highlight the importance of the A8 segment in this process. We propose that A8 cells affect the development of structures originated from A9 and A10 through the activity of the PVF1 protein diffusing from A8. Although our data concern transcript expression, Rosin et al., 2004, demonstrated that PVF1 is capable of extensive lateral diffusion, so it has the properties of a long range signaling molecule.

PVFs could form homo and heterodimers, what opens the possibility of different effects in the binding responses of the receptor. McDonald et al., 2003, observed that homodimers are not equivalent, because PVF1 seems to be the relevant signal for the migration of border cells and Brüncker et al., 2004, describe two function for PVR in the embryonic hemocytes, suggesting a diversity of functions. We have not analyzed other PVFs and although we observed some partial redundancy, it will be necessary to separate PVF individual or associated functions. 

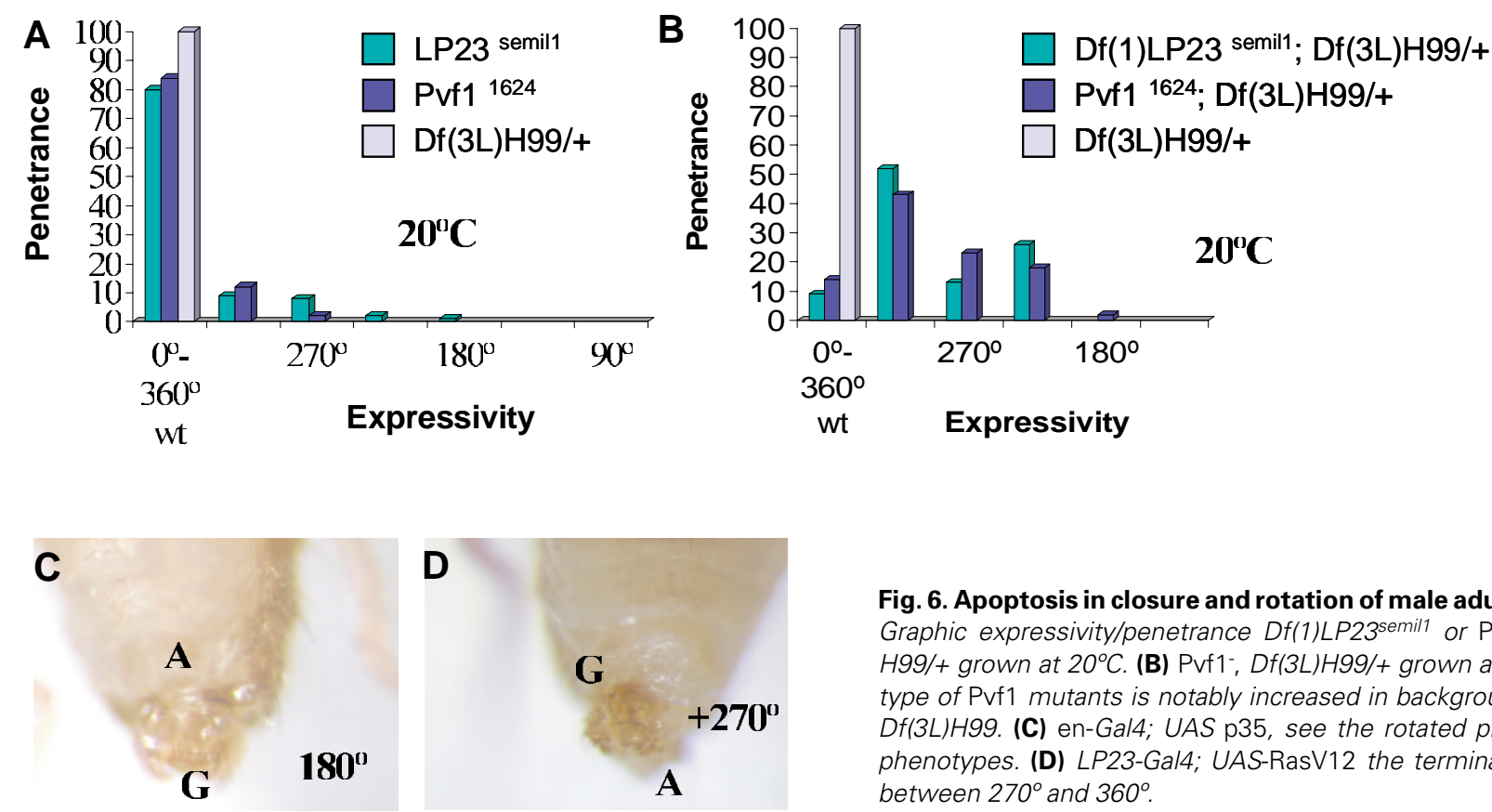

Fig. 6. Apoptosis in closure and rotation of male adult terminalias. (A) Graphic expressivity/penetrance Df(1)LP23 ${ }^{\text {semill }}$ or Pvf1 ${ }^{1624}$ and $D f(3 L)$ $\mathrm{H} 99 /+$ grown at $20^{\circ} \mathrm{C}$. (B) Pvf1- Df(3L)H99/+ grown at $20^{\circ} \mathrm{C}$. The phenotype of Pvf1 mutants is notably increased in background hemizygous to Df(3L)H99. (C) en-Gal4; UAS p35, see the rotated plus the split dorsal phenotypes. (D) LP23-Gal4; UAS-RasV12 the terminalia is miss-rotated between $270^{\circ}$ and $360^{\circ}$.

We obtained indirect evidence about where the PVR receptor is activated or expressed. First, by recognition of factors that mediate the activity of the PVF1/PVR signaling mechanism (i.e. $\mathrm{dpERK}$ ), whose expression was located at the periphery of the group of cells expressing Pvf1. Second, blocking PVF1 activity using $P v P N$ and overexpressing $P v f 1$ the effects are stronger in the engraileddomain where $P v f 1$ is not expressed. These findings provide additional evidence that there are specific domains for ligand expression and for responsive cells. In the ovary Pvf1 is expressed in the ovule while Pvris expressed in the follicle cells, the importance of this non overlapping domains is reflected by the fact that overexpression of a constitutive active form of the receptor ( $\lambda$ Pvr) produces the same phenotype of its lack of function (Duchek et al., 2001). In the wing disc Rosin et al., 2004 observed that the restrictions in the activity are regulated by a polarized secretion of the ligand in the apical membrane.

\section{Apoptotic genes and the JNK pathway}

Mutations in the pro-apoptotic gene hid have been shown to affect male terminalia rotation (Abbot and Lengyel, 1991), although this phenotype was observed in trans heterozygotes for $D f(3 L) H 99$, which includes the three pro-apoptotic genes hid, rpr and grim (Grether et al., 1995). Trans heterozygotes for hidmutations are of wildtype phenotype, indicating that the rotated phenotype over deficiency is not only due to hidbut to the haploinsufficiency of one or the two other genes. Our result that preventing cell death with p35 leads to miss rotation and split dorsal is also consistent with an involvement of apoptosis in these processes.

Additionally, we show that overexpressing puc results in the same phenotypes as PVF1/PVR and reduction of apoptosis, lowering puc rescues the rotated terminalia defects observed in DfH99/ + males. The level of pucis considered as indicative of the JNK pathway activity (Martín-Blanco et al., 1998; Moreno et al., 2002 a-b) so these experiments suggests that JNK promotes apoptosis, probably by up-regulating hid.

\section{PVF1/PVR, JNK and apoptosis}

The fact that alterations in the PVF1/PVR pathway and in JNK/ apoptosis give rise to similar phenotypes suggests a functional link between these two pathways.

The penetrance of the phenotypes of Pvf1 mutations in the terminalia increases when they are additionally heterozygous for Df(3L)H99. This increase is non additive, suggesting PVF1/PVR and the apoptotic machinery affect the same aspect of the process. The overexpression of Pvf1 ectopically activates puc and impedes the normal rotation and closure. This activation would down-regulate the JNK apoptotic pathway (Martín-Blanco et al., 1998), thus reducing apoptosis and giving rise to the terminalia phenotype, but since the JNK pathway is a transcriptional activator of puc (Martín-Blanco et al., 1998), this result opens up the possibility that Pvf1 ectopically activates JNK rather than puc.

Apoptosis is necessary for terminalia rotation and dorsal closure and our results and those of others indicate that it is mediated by JNK activity. PVF1/PVR is also affecting these processes and our data suggest that PVF1/PVR may also affect JNK-mediated apoptosis. It is not clear however, whether all these elements act on the same developmental cascade.

\section{Materials and Methods}

\section{Fly stocks}

Mutant Pvf1 alleles used in this study are: $P v f 1^{1624}$ and EP11235, corresponding to EP insertions in the Pvf1 gene (Rørth, 1998; Duckek et al., 2001). Two deficiencies in $P v f 1, D f(1) \angle P 23^{\text {semil1 }}$ and $\angle P 23^{\text {semil2 }}$, were isolated in this work and are described in the text. Dp(1;Y)W39 (Prado et al., 1999) is a duplication covering the Pvf1 gene. The Gal4/UAS system (Brand and Perrimon, 1993) was used to drive the expression of several gene constructs: UAS-Pvf1 expressing the long spliced form of the Pvf1 gene (Duchek et al., 2001); UAS-Pvrand UAS-PVPDN express the native and a dominant negative form of the receptor for the PVF proteins, respectively (Duchek et al., 2001); UAS-Ras ${ }^{V 12}$ (Fontini et al., 1992) 
A
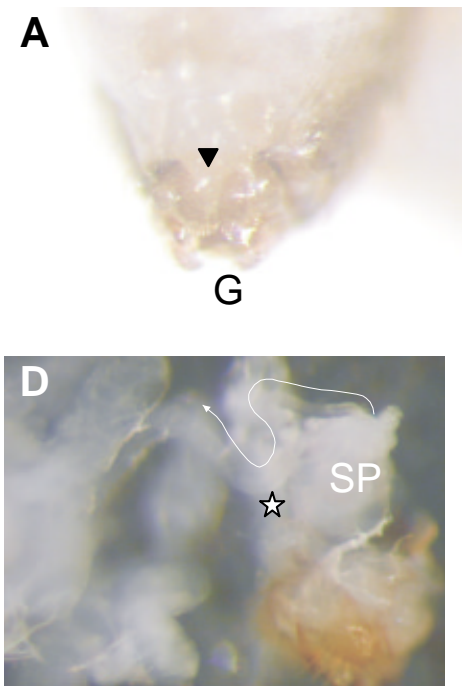
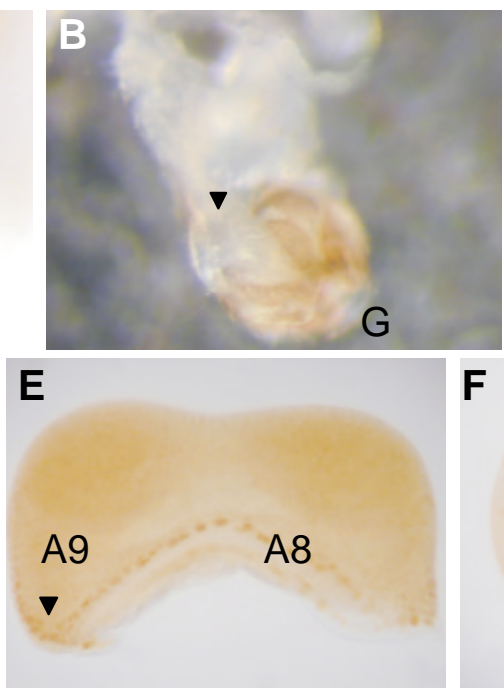

C

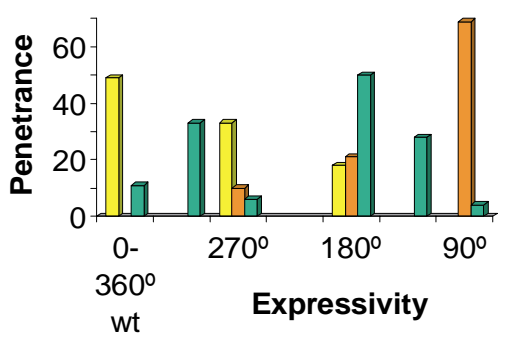

$\mathbf{F}$

$\square$ Dpp-Gal4:UAS puc $\square$ Ptc-Gal4:UAS puc $\square$ En-Gal4:UAS puc

puc. These experiments were performed at $24^{\circ} \mathrm{C}$ because dpp and ptc driven expression at $30^{\circ} \mathrm{C}$ is a lethal conditic phenotype with the targeted expression in the en-ptc domains. (D) Dissected en-Gal4; UAS-puc male. The external terminalia was at $180^{\circ}$, the white line follows the spermiduct, the duct does one bend (white star) and the sperm pump is on the left side of the fly. (E,F) Male genital discs. Expression of puc by puc-lacZ transgene revealed by anti- $\beta$ gal stain. The insertion of $P$-lacZ in puc generates a recessive lethal mutation called puc ${ }^{E 69}$. In males, puc ${ }^{E 69}$. puc ${ }^{E 69}$ ,+ wt puc expression is in A8 cells which surround the segment and extend to A9. (F) hh-Gal4; UAS-Pvf1/puc E69, stained with anti-Bgal to reveal puc expression, the ectopic expression of Pvf1 activates the gene puckered outside its own domain, the arrows point the normal and the ectopic expression.

expresses a constitutive form of the RAS protein, while UAS- puc expresses the wild type allele of the puckered (puc) gene (Martín-Blanco et al., 1998). The driver's dpp-Gal4, en-Gal4, ptc-Gal4 and hh-Gal4 (Staehling-Hampton, K. et al., 1994; Hinz, U. et al., 1994; Tanimoto, H. et al., 2000).), as well as the hid allele hid422 and $\mathrm{Df}(3 \mathrm{~L}) \mathrm{H} 99$ (Abbott and Lengyel, 1991; Grether et al., 1995), have previously been described. $p c^{E 69}$ is a null allele for the gene puccarrying an insertion of a lacZgene (Martín- Blanco et al., 1998).

\section{Immunostaining, $X$-Gal staining and in situ hybridization}

Immunostaining and $X$-Gal staining were performed as described (Macías et al., 1990). The anti dpERK used is a monoclonal anti-Map Kinase, Activated antibody (SIGMA \#M8159). The detection system used for the immunoreactions was ELITE of Vector Lab. The RNAs probes were transcribed from: Pvf1: EST 30334; CG7101: EST 44815. For in situ hybridization we followed the protocol adapted from Lehner and O'Farrell (1990). Stained embryos or discs were soaked in $87 \%$ glycerol, mounted onto cover slips and photographed with a NIKON digital camera Coolpix 950. The images were obtained with a stereomicroscope LEICA MZ 9.5 and a NIKON LABOPHOT light microscope. Given the low amount of products to be analyzed in these studies, incubation and developing times in our protocols were adjusted to optimize signal/background ratios.

\section{PCR and Southern analysis}

The LP23-Gal4 insertion was located by inverse PCR (Rehm, J.E; BDGP Resources). The primers used to map the LP23-Gal4 site of insertion are: 5'-CTG AGC GAC ATC TCA CGT CC-3' and 5'-TAG TCA GCG GAG ACC TTT TGG-3' for the 5' end and 5'-TGA CCA TGA TTA CGC CAA GCG-3' and 5'-CCA AGC ACC AGC AAG TTC CG-3' for the 3' end. For Southern analyses, genomic DNA from FM7 ( $w t$ control), Pvf1 1624 and EP11235 (insertions into Pvf1), Df(1)LP23semil1, $\mathrm{Df}(1) \mathrm{LP} 23^{\text {semil2 }}$ and LP23-Gal4 were digested with the restriction enzymes Pvill, HindIII, Bghl, Xhol and EcoRI. Blots were hybridized with $\alpha-\left({ }^{32} \mathrm{P}\right)$-dATP (Amersham) oligolabeled probes (Feinberg and Vogelstein, 1984) prepared from template DNA corresponding to EST 30334, EST
44815, pBluescript KS and the Ga/4gene. EST 30334 is a near full-length cDNA of Pvf1 (Cho et al., 2002).

\section{Acknowledgements}

We thank Sigrid Baars, Susana Romani, Ana Carmena, Gladys Sala, Silvia Capisano, Graciela Macias and Antonio Sánchez for their valuable help, Ernesto Sánchez-Herrero for critically reading the manuscript and the anonymous reviewers for constructive comments. We also thank Andrea Brand and members of her lab for plasmids. Pernille Rorth, Herman Steller, D.A. Wassarman, Sonsoles Campuzano and the Bloomington Stock Center for sending stocks. Special thanks to Kathy Mattews for her advice with flies stocks. This work has been supported by grants from the Fondo Nacional de Ciencia y Tecnologia (FONCYT, Argentina) to A.M and A.L.R. and the Third World of Academic Science (TWAS) to A.M. A.M. and A.L.R. are supported by CONICET, Argentina.

\section{References}

ADAM, G., PERRIMON, N. and NOSELLI, S. (2003). The retinoic-like juvenile hormone controls the looping of left-right asymmetric organs in Drosophila. Development 130: 2397-2406.

ABBOTT, M.K. and LENGYEL, J. (1991). Embryonic head involution and rotation of male terminalia require the Drosophila locus head involution defective. Genetics 129, 783-789.

BERGMANN, A., AGAPITE, J., MCCALL, K. and STELLER, H. (1998). The Drosophila Gene hid is a direct molecular target of Ras-dependent survival signaling. Cel/95: 331-341.

BRAND, A. H. and PERRIMON, N. (1993). Targeted gene expression as a means of altering cell fates and generating dominant phenotypes. Development 118: 401-415.

BRÜCKNER, K., KOCKEL, L., DUCHECK, P., LUQUE, C.M., RØRTH, P. and PERRIMON, N. (2004). The PDGF/VEGF receptor controls blood cell survival in Drosophila. Dev. Cel/7: 73-84.

CALLEJA, M., MORENO, E., PELAZ, S. and MORATA, G. (1996). Visualization of gene expression in living adult Drosophila. Science 274: 252-255. 
CASANOVA, J., SANCHEZ-HERRERO, E. and MORATA, G. (1986. Identification and characterization of a parasegment specific regulatory element of the Abdominal-B gene of Drosophila. Cel/47: 627-636.

CHAO, S.H. and NAGOSHI, R.N. (1999). Induction of apoptosis in the germ line and follicle layer of Drosophila egg chambers. Mech. Dev. 88: 159-172.

CHEN, P., NORDSTROM, W., GISH, B. and ABRAM, J.H. (1996). Grim, a novel cell death gene in Drosophila. Genes Dev. 10: 1773-1782.

CHO, N-K., KEYES, L., JOHNSON, E., HELLER, J. RYMER, L., KARIM, F. and KRASNOW M.A. (2002). Developmental control of blood cell migration by the Drosophila VEGF pathway. Cel/108: 865-876.

DUCHEK, P., SOMOGYI, K., JÉKELY, G., BECCARI, S. and RØRTH, P. (2001). Guidance of cell migration by the Drosophila PDGF/VEGF receptor. Cell107: 17-26.

FAHMY and FAHMY (1958). New mutant report. D.I.S. 32: 67-78.

FEINBERG, A.P. and VOGELSTEIN, B. (1984). A technique for radiolabeling DNA restriction endonuclease fragments to high specific activity». Anal Biochem. 137: $266-7$

FONTINI, M.E., SIMON, M.A. and RUBIN, G.M. (1992). Signaling by the Sevenless protein tyrosine kinase is mimicked by Ras 1 activation. Nature 355: 559-561.

GABAY, L., SEGER, R. and SHILO, B-Z. (1997). In situ activation pattern of Drosophila EGF Receptor pathway during development. Science 277: 11031106.

GLEICHAUF, R. (1936). Anatomie und Variabilitat des Geschlechtapparates von Drosophila melanogaster (Meigen). Z.Wiss.Zool. 148:1-66.

GLOOR, G.B., NASSIF, N.A., JOHNSON-SCHLITZ, D.M., PRESTON, C.R. and ENGELS, W.R. (1991). Targeted Gene Replacement in Drosophila via P element-induced gap repair. Science 253: 1110-1117.

GRETHER, M.E., ABRAMS, J.M., AGAPITE, J., WHITE, K. and STELLER, H. (1995). The head involution defective gene of Drosophila melanogasterfunctions in programmed cell death. Genes and Development 9: 1694-1708.

HAY, B.A., WOLFF, T. and RUBIN, G.M. (1994). Expression of baculovirus p35 prevents death in Drosophila. Development 120: 2121-2129.

HEINO, T.P., KÄRPÄNEN, T., WAHLSTRÖM, G., PULKKINEN, M., ERIKSSON U., ALITALO, K. and ROOS, C. (2001). The DrosophilaVEGF receptor homolog is expressed in hemocytes. Mech. Dev. 109: 69-77.

HELDIN, C.H. and WESTERMARK, B. (1999). Mechanism of action and in vivo role of platelet-derive growth factor. Physiol. Rev. 79: 1283-1316.

HINZ, U., GIEBEL, B. and CAMPOS-ORTEGA, J.A. (1994). The basic-helix-loophelix domain of Drosophila lethal of scute protein is sufficient for proneural function and activates neurogenic genes. Cel/76: 77-87.

KURADA, P. and WHITE K. (1998). Ras promotes cell survival in Drosophila by downregulating hid expression. Cel/95: 319-329.

LEHNER, C.F. and O'FARRELL, P.H. (1990). The roles of Drosophilacyclins A and $\mathrm{B}$ in mitotic control. Cel/61: 535-547.

MACIAS, A., CASANOVA, J. and MORATA, G. (1990) Expression and regulation of the $a b d-A$ gene of Drosophila. Development 110: 1197-1207.
MARTIN-BLANCO, E., GAMPEL, A., RING, J., VIRDEE, K., KIROV, N., TOLKOVSKY, A.M. and MARTINEZ-ARIAS, A. (1998). Puckered encodes a phosphatase that mediates a feedback loop regulating JNK activity during dorsal closure in Drosophila. Genes Dev. 15: 557-570.

MCDONALD, J.A., PINHEIRO, E.M. and MONTELL, D.J. (2003). PVF1, a PDGF/ VEFG homolog, is sufficient to guide border cells and interacts genetically with Taiman. Development 130: 3469-3478.

MORENO, E., BASLER, K. and MORATA, G. (2002)a. Cell competes for decapentaplegic survival factor to prevent apoptosis in Drosophila wing development. Nature 416: 755-759.

MORENO, E., YAN, M. and BASLER, K. (2002)b. Evolution of TNF signaling mechanisms: JNK-Dependent Apoptosis Triggered by Eiger, the Drosophila homolog of the TNF Superfamily. Curr. Biol. 12: 1263-1268.

MUNIER, A-I., DOUCET, D., PERRODOU, E., ZACHARY, D., MEISTER, M., HOFFMANN, J.A., JANEWAY, C.A. and LAGUEUX, M. (2002). PVF2, a PDGF/ VEGF growth factor, induces hemocytes proliferation in Drosophila larvae. EMBO reports 3: $1195-1200$

PRADO A., CANAL, I. and FERRUS, A. (1999). The haplolethal region at the $16 \mathrm{~F}$ gene cluster of Drosophila melanogaster. structure and function. Genetics 151: 163-175.

RORTH, P. (1998). Gal4 in the Drosophila female germline. Mech. Dev. 78: 113 118

ROSIN, D., SCHEJTER, E., VOLK, T. and SHILO, B-Z. (2004). Apical accumulation of the Drosophila PDGF/VEGF receptor ligands provides a mechanism for triggering localized actin polymerization. Development 131: 1939-1948.

SANCHEZ-HERRERO, E. and CROSBY, M (1988). The Abdominal- $B$ gene of Drosophila melanogaster. overlapping transcripts exhibit two different spatial distributions. EMBO J7: 2163-2173.

SANCHEZ, L. and GUERRERO, I. (2001). The development of Drosophila genita disc. BioEssays 23: 698-707.

SEGER, R. and KREBS, E.G. (1995). The MAPK signaling cascade. The FASEBJ. 9: 726-735.

STAEHLING-HAMPTON, K., JACKSON, P. D., CLARK, M. J., BRAND, A. H. and HOFFMANN, F. M. (1994). Specificity of bone morphogenetic protein-related factors: Cell fate and gene expression changes in Drosophila embryos induced by decapentaplegic but not 60A. Cell Growth Diffn 5: 585-593.

TANIMOTO, H., ITOH, S., TEN DIJKE, P. and TABATA, T. (2000). Hedgehog creates a gradient of DPP Activity in Drosophila wing imaginal discs. Mol. Cell 5: 59-71.

WASSARMAN, D.A., AOYAGI, N., PILE, L.A. and SCHLAG, E.M. (2000). TAF250 is require for multiple developmental events in Drosophila. Proc. Natl. Acad. Sci. USA 97: 1154-1159.

Received: June 2004

Reviewed by Referees: July 2004

Modified by Authors and Accepted for Publication: September 2004 